\title{
The use of occlusal splints in temporomandibular disorders - an overview.
}

\author{
Andreea Kui ${ }^{1}$, Silvia Pop ${ }^{2}$, Smaranda Buduru ${ }^{1}$, Marius Negucioiu ${ }^{1}$ \\ ${ }^{1}$ Iuliu Hatieganu University of Medicine and Pharmacy, Cluj-Napoca, Romania \\ ${ }^{2}$ George Emil Palade University of Medicine, Pharmacy, Science, and Technology of Targu Mures, Romania
}

\begin{abstract}
Temporomandibular disorders (TMD) affect the temporomandibular joints, the masticatory muscles, and surrounding tissues. Among symptoms such as jumps, joint noises, reduced mouth opening (closed lock), difficulties in closing the mouth (subluxation or open lock), pain is the most common symptom encountered among patients diagnosed with temporomandibular disorders. As literature on this topic is abundant and sometimes controversial, the authors focus on reviewing the state of art of occlusal splints indications. Therefore, the most common occlusal splints, like Lucia jig, nociceptive trigeminal inhibition (NTI), directive splints, etc., are being described, based on their design and therapeutic indications. Cases of malocclusions associated or not with parafunctions are usually manageable using the splints mentioned in this article. In case of disc displacements, occlusal appliances can be used, but as the etiology is multifactorial, there are some limitations, depending on the complexity of each clinical situation.
\end{abstract}

Keywords: occlusal splints, NTI, Jeanmonod anterior bite-plane.

\section{Introduction.}

Temporomandibular disorders (TMD) represent a real challenge in everyday practice due to the high complexity of different aspects such as diagnostic methods or treatment options [1]. Temporomandibular disorders refer to a group of musculoskeletal and neuromuscular conditions, affecting the temporomandibular joints and the masticatory muscles as well as the surrounding tissues. Pain is the most common symptom encountered among patients diagnosed with TMD [2], but other symptoms, including joint jumps, joint noises, reduced mouth opening (closed lock), difficulties in closing the mouth (subluxation or open lock) may often be encountered as well [3].

There are several treatment options in case of temporomandibular disorders: occlusal equilibration (adjustments), drug therapy, occlusal splint therapy, surgical techniques, physiotherapy (laser therapy, ultrasound, TENS, acupuncture, etc.), patient's education (associated with behavior therapy). Often, a combination of several types of treatment options is used in the management of TMD [3].

Occlusal splints are intraoral appliances used for diagnosis or treatment, for occlusal stabilisation, dental wear prevention, or in case of temporomandibular disorders (muscle relaxation or joint stabilization), as they affect the relationship of the mandible to the maxillae [3].

The extensive number of studies published so far proves that occlusal splints are widely used today in the management of TMD. Nevertheless, because various splint designs are available, it is important to analyze the different outcomes which could influence the clinical decision $[4,5,6]$.

This article presents the types of occlusal splints available, based on their characteristics, aiming to provide helpful information for clinical practitioners in treating patients suffering from temporomandibular disorders.

Why an occlusal splint?

Occlusal splints are commonly used in the management of patients with temporomandibular disorders (TMD) as their main effect is muscle relaxation [7]. Those appliances aim to protect the temporomandibular joint (TMJ) discs from dysfunctional forces, preventing any permanent displacements or even disc perforations. There are also additional goals in splint therapy, such as preventing muscle parafunctions and relieving associated pain by maintaining a stable and balanced occlusion $[8$, 9]. 
What types of occlusal splints are available?

In daily practice, the information gathered during clinical and para-clinical examinations along with the differential diagnosis will influence the decision process regarding the prescription of a particular type of splint. In this matter, understanding the effects of each splint will also guide the decision-making [10].

Several types of occlusal splints have been developed over the years. For a better understanding they have also been classified into two different categories, depending on their design: (1) permissive splints - anterior midpoint contact permissive splint, and full contact permissive splint, (2) anterior repositioning directive splint $[7,10]$.

Permissive splints have classic designs and their role is to eliminate the abnormal occlusal contacts as well as to determine a balanced muscle function (by reducing parafunctional activity). The principle behind a permissive splint is that by altering the occlusion, teeth will no longer interfere with complete seating of the condyles, this way the muscle activity can also be controlled [8].

1. Anterior midpoint contact permissive splints have a design which allows the disocclusion of all teeth, except the incisors. Among those types of splints, the most popular are Lucia jig (Great Lakes Orthodontics, LTD, Tonawanda, NY), nociceptive trigeminal inhibition (NTI), B splint (Bruxism), or Hawley anterior biteplate [8].

The anterior midpoint contact permissive splints are believed to determine the release of the lateral pterygoid muscle on closure, as well as neck positioning muscles, to remove the occlusal interferences, and to allow the mandibular condyles freedom for full seating during the closure [11].

While a classic anterior midpoint stop appliance can be fabricated in the office (like Lucia jig), NTI devices and B-splints are commercially available and then customized for fit and occlusion directly in the patient's mouth. When using NTI splints, only 1 or 2 lower anterior teeth should touch the occlusal platform, which will lead to reflexive relaxation of the masticatory muscles. Nevertheless, there are some disadvantages in using anterior midpoint stop appliances, which imply occlusal changes, including intrusion or proclination of the incisors, or even open bite $[12,13]$.

The Hawley anterior biteplate will cover the 6 anterior teeth +/- the first premolars which may offer a better stability in both anterior and posterior areas of occlusion. However there should be strict limitation of only nighttime wear. The Hawley device may also be indicated when tooth clenching (associated with myofascial pain) determines the breaking of a full coverage appliance [12].

Jeanmonod anterior bite-plan (figure 1) covers the 6 anterior maxillary teeth and it is designed as a palatal-coverage horseshoe shape with an occlusal table. It has the ability to prevent clenching, as the posterior teeth will no longer be engaged in functional or parafunctional activities. The result is the muscle relaxation, as well as pain reduction $[14,15]$.

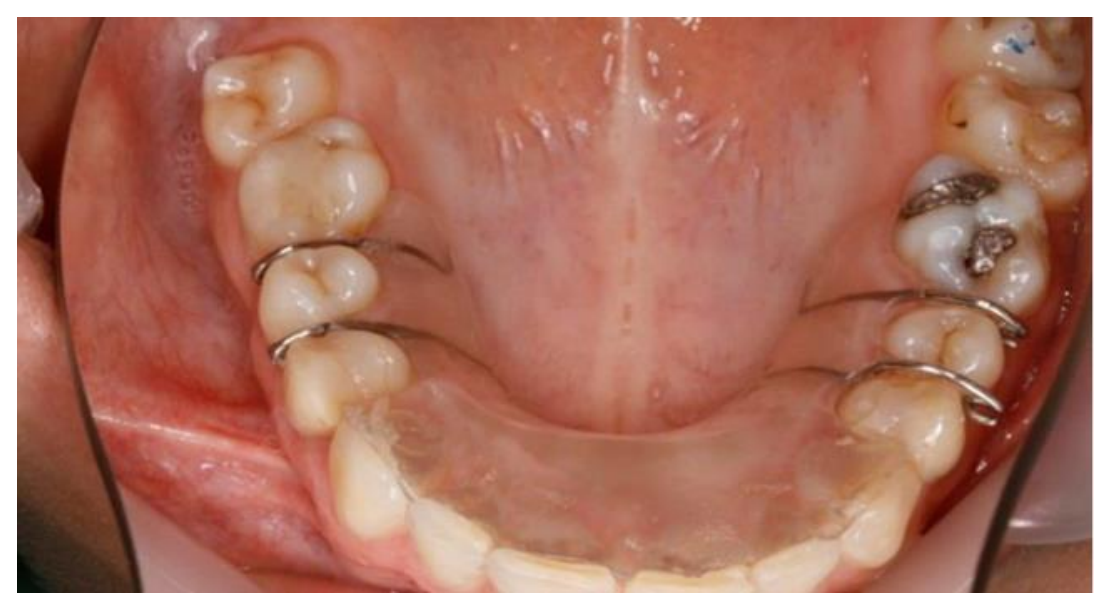

Figure 1. Jeanmonod anterior bite-plane 
Full contact permissive splints' design allow to create an ideal occlusion, but in a reversible manner. The occlusion on the splint should be adjusted in centric relation, this way it provides joint stabilisation. An anterior ramp will provide the anterior guidance, while all posterior teeth will disocclude immediately, in order to reduce the elevator muscle activity [10].

One of the most common full contact permissive splints is Michigan splint. It is usually indicated on the maxillary arch, but for phonetic and aesthetic reasons, it can also be placed in the mandibular arch [3]. Among other advantages of lower full contacts permissive splints we can also mention a better patient compliance, when the instructions indicate to wear the splint during daytime as well as nighttime, or even less tooth discomfort when the retention is assured only by the lingual surfaces of the lower posterior teeth [10].

Full contact permissive splints will eliminate any discrepancies between seated joints (in centric relation - CR) and occlusion (during maximum intercuspal position - MI), and will offer the opportunity to observe occlusion and joint stability over time, distribute the occlusal forces evenly while eliminating the occlusal interferences [11].

2. Anterior repositioning directive splints have the purpose to guide the condyles away from the fully seated joint position, in situations when joint pain is an issue. The mandible is guided into a forward posture on closure into the occlusal splint [10]. This is the reason why those types of splints are useful in two clinical situations: severe trauma with retrodiscal edema, and in case of chronic painful disk displacements $[10,11]$.

Apart from the two types of splints mentioned above, there are also other types of splints available, such as posterior bite plane appliance, pivot appliance, and pseudo permissive splints (e.g soft splints).

Posterior bite plane splints are applied on the lower arch, and the design consists of a bilateral hard acrylic resin table connected through a lingual metal bar, positioned only on the posterior teeth, and creating the disocclusion of the anterior teeth. Although there is no significant scientific evidence, some authors reported that this type of splint may enhance the athletic performance, increasing the physical strength. This type of splint is also indicated in cases of severe loss of vertical dimension of occlusion or when major changes are required in anterior positioning of the mandible [3, 11].

Pivoting appliances are hard devices, manufactured either on the maxillary or mandibular arch, with a single posterior occlusal contact, and placed as distally as possible, on each hemi-arch. Those appliances are known to reduce the pressure inside TMJ, as the mandible fulcrums around the pivot, unloading the joints. Unilateral pivot appliance is a modified version of the pivot appliances, having only a unilateral occlusal contact, placed as posterior as possible. This way, when the mouth closes, the pivot will load the contralateral TMJ and slightly unload the ipsilateral joint $[3,16]$.

Pseudo permissive splints, like soft and resilient splints or hydrostatic splints (Aqualizer $\AA$ ) are fabricated from resilient materials. Soft rubber splints cannot balance the occlusal contacts, this is why they do not provide the characteristics necessary for successful splint therapy and can be used mainly as mouth guards. They are usually placed on the maxillary arch [8].

A hydrostatic splint, Aqualizer $\AA$, is known to immediately improve biomechanics, due to its unique water system. Several features of this appliance should be mentioned: it supports the mandible in a comfortable position, eliminates abnormal tooth contacts, straightness the bite and enables systemic function and balance, while allowing the whole body to naturally balance itself $[17,18,19]$.

Aqualizer $\AA$ was originally developed by Lerman, and it consists in a bilateral waterfilled plastic chamber attached to an acrylic palatal appliance. This way, the lower teeth will occlude on water filled chambers [3].

AquaSplint ${ }^{\circledR}$ (figure 2) is a pre-fabricated appliance which can be customized and selfadjusted; two water pads united together by a tube represent the pre-fabricated splint, while the acrylic saddle can be relined with a longterm silicone material, providing individualization. It offers stability and comfort, while immediately reducing the pain, 
and offering a hydrostatic balance between the two arches, because of the two water pads [20].

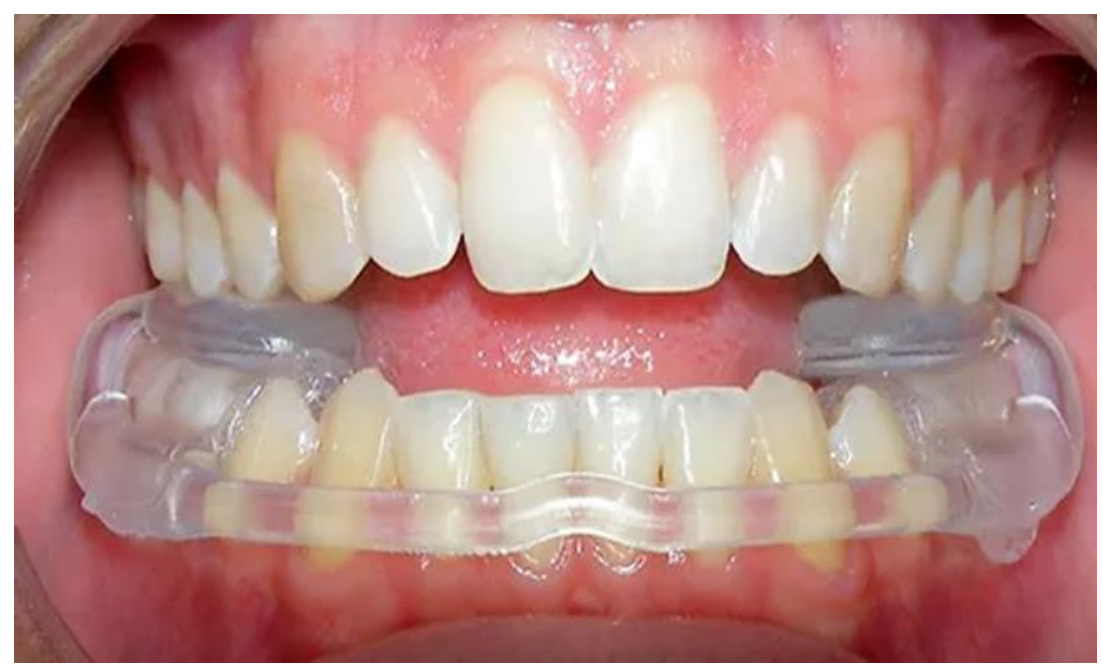

Figure 2 - AquaSplint ${ }^{\circledR}$ appliance [20]

When to indicate splint therapy and which type of splint?

Splint therapy should be indicated in order to obtain masticatory muscle relaxation, to allow the mandibular condyles to seat comfortably in centric relation, or to protect dental units from bruxism or other parafunctions.

In case of temporomandibular disorders, the indication of a certain type of occlusal appliance will be according to the disorder's type - muscle disorder (Type I) / joint disorder without inflammation (Type II - disc displacement) or with inflammation (Type III) $[10,12]$.

In a research published in 2018, Greene synthesises that, no matter the type of TMD, as an initial therapy, a full contact permissive appliance should be taken into consideration. Then, if symptoms persists or they aggravate, different approaches should be considered: for type I disorders, refractory to the initial splint therapy (full contact appliance), an anterior midpoint contact appliance (either Lucia jig, NTI or Hawley device) can be used until the symptoms disappear; afterwards, when the patient becomes asymptomatic, the appliance should be changed with the initial one - full contact permissive splint [12].

For type II temporomandibular disorders, in case of patients presenting symptoms even after full contact permissive splint, a directive splint should be considered for 2 to 3 months, and afterwards it is indicated to return to the initial appliance [12].

In case of type III disorder, the same approach is indicated: a full contact permissive appliance, but in cases with severe inflammation, a directive splint can be used for 1 to 2 months, or until the inflammation is reduced; afterwards, the initial splint can be reused [12].

Dawson's approach regarding the treatment options refers to the symptoms' complexity, from simple scenarios to more complex ones.

In cases with simple occluso-muscle problems, where there are minimal signs of disorder such as tooth wear, tooth mobility, muscle tenderness during palpation, occlusal instability and uncoordinated muscle activity (due to interference between centric occlusion and maximum intercuspal position), Dawson's approach would be the wear of an anterior midpoint contact permissive splint (Lucia Jig, NTI, etc.) for 1 to 5 minutes/day, and occasionally, for several hours during nighttime (when total relaxation of the masticatory muscles is required)[10, 21].

When the occluso-muscle issues are associated with parafunctions (bruxism, clenching, or grinding) there will be symptoms with a higher impact on the quality of life, with headaches, sore teeth, muscle pain, tooth wear facets, etc. In these situations, in order to resolve the pain, anterior midpoint contact permissive splints should be worn 24 hours per 
day (except during mealtimes), between 1 to 4 weeks. Among the splints indicated in such situations are $\mathrm{B}$ splints (which can be performed in the office) or NTIs (prefabricated and relined with acrylic materials). In a short period of time (2 to 4 weeks), those types of splints proved to be effective, but therapy should be continued with proper occlusal correction after a complete occlusal analysis. After this phase, the equilibrated occlusion with proper anterior and lateral guidance, without interferences, can be maintained by using a full arch permissive splint. Dawson also believes that in order to prevent the negative effects of nocturnal parafunctions, splints should be worn during nighttime as well, ideally dual splints covering both arches [10, 22, 23].

In case of disk displacement with reduction, the use of full arch permissive splints should be effective; the duration of the treatments should last between 6 to 8 weeks. If the disk displacement is complete (without reduction) then the same type of splint (full arch permissive appliance) would be indicated for a minimum of 3 months. In this situation, occlusion on the splint should be adjusted weekly, until it stabilizes $[10,11]$.

Some authors suggest an anterior repositioning directive splint (for a short period of time -2 weeks), if joints cannot not be seated fully without discomfort (inflammation of the joint), followed by a full arch permissive splint (for 3 months or even more) $[10,11]$. But this aspect is controversial, as some patients accuse an exacerbation of pain when treated with directive splints.

What characteristics should a splint meet, for functional consideration?

A splint should assure stable occlusal contacts with the antagonist teeth, preferably in centric relation; it should provide immediate posterior disocclusion by the anterior and condylar guidance; the splint should be adjusted continuously, and it should offer freedom in movement for neuromuscular harmony; also, the splint should not negatively affect the aesthetics, and the patient should be comfortable wearing the splint $[11,23,24]$.
What would be the limitations of splint therapy?

Several studies published underline the complications that may occur in case of extended use of segmental appliances. The most common complications refer to intrusion of the teeth covered by the splint or in contact with it, and the extrusion of the teeth not touching the appliance. For preventing those complications, the practitioner should consider indicating the patient either a full arch permissive splint, or dual splint covering both arches, during nighttime $[25,26]$. There is also some evidence suggesting that anterior midpoint contact permissive splints might increase the risk of further disk displacement [10].

Additionally, the development of digital techniques (CAD/CAM and 3D printing) for performing occlusal splints offers several advantages, like perfectly adjusting the splint's thickness, time-efficiency, the possibility of duplication at any moment [27].

\section{Conclusion}

Splint therapy is an effective method of treatment in case of temporomandibular disorders. But special attention should be paid on establishing a correct and complete diagnosis, as signs and symptoms will vary, depending on the type of temporomandibular disorder.

Cases of malocclusions associated or not with parafunctions are usually manageable using the splints mentioned in this article, but periodic check-ups are needed in order to prevent further complications. In case of disc displacements, occlusal appliances can also be used, but as the etiology is multifactorial, there are some limitations, as creating long term joint stability is questionable.

Conflict of interest: None declared.

All authors contributed equally to this article.

\section{References}

1. Garrigós M, Elizagaray-García I, Domínguez AA, Del-castillo JL, Gil-Martinez A. Temporomandibular disorders:improving outcomes using a multidisciplinary approach. J MultidiscipHealthc. 2019;12:733-747. 
2. Conti PCR, Da Mota Correa AS, Lauris JRP, Stuginski-Barbarosa J. Management of painful temporomandibular joint clicking with different intraoral devices and counseling: a controlled study. J Appl Oral Sci. 2015;23(5):529-535.

3. Alqutaibi AY, Aboalrejal AN. Types of Occlusal Splint in Management of Temporomandibular. J Arthritis. 2015;4(4):176.

4. Fricton J, Look JO, Wright E, et al. Systematic review and meta-analysis of randomized controlled trials evaluating intraoral orthopedic appliances for temporomandibular disorders. J Orofac Pain. 2010;24(3):237-254.

5. Gray RJM, Davies SJ, Quayle AA. Temporomandibular Disorders: A Clinical Approach. London: British Dental Association;1995.

6. Ebrahim S, Montoya L, Busse JW, Carrasco-labra A, Guyatt $\mathrm{GH}$. The effectiveness of splint therapy. J Am Dent Assoc. 2012;143(8):847-857.

7. Mishra A, Mahajan S, Tandon R. A-Z of Occlusal Splints in Orthodontics - Part II. Heal Talk, Orthod. 2018;10(6):59-60.

8. Bali SK, Naqash TA, Rajput G. Occlusal Splint Therapy: An Overview. Univ J Dent Sci. 2015;1(1):82-84.

9. Yadav S, Karani JT. The Essentials Of Occlusal Splint Therapy. Int J Prosthetic Dent. 2011;2:12-21.

10. Dawson PE. Functional Occlusion: From TMJ to Smile Design. St. Louis, MO: Mosby; 2007.379-392.

11. Jagtap A, Shadakshari S, RaghavendraSwamy K, Bora N. Occlusal Splint Therapy for a Dysfunctional Temporomandibular Joint. J Orofac Res. 2014;4(2):115-117.

12. Hasanoglu Erbasar GN, Alpaslan C, EroglUInan G. Can an NTI-tss device be effective as a first-line therapy in patients with TMD myofascial pain ? J Oral Rehabil. 2017;(9):1-5.

13. Aksakalli S, Temucin F, Pamukcu A, Ezirganlı S, OguzKazancioglu $\mathrm{H}$, Malkoc MA. Effectiveness of two different splints to treat temporomandibular disorders. J OrofacOrthop. 2015;76(4):318-327.

14. Seifeldin SA, Elhayes KA. Soft versus hard occlusal splint therapy in the management of temporomandibular disorders ( TMDs ). Saudi Dent J. 2015;10(2):1-6.

15. Yeshwante B, Choudhary N, Kadam P. Occlusal Splint Therapy. Ann Dent Spec. 2015;3(4):100-102.

16. Dodeja DL, Singh R, Mistry G. Splints: Decoded. IOSR J Dent Med Sci. 2019;18(5):11-19.
17. Amin A, Meshramkar R, Lekha K. Comparative evaluation of clinical performance of different kind of occlusal splint in management of myofascial pain. J Indian Prosthodont Soc. 2016;16( 2):176181.

18. Srivastava R, Jyoti B, Devi P. Oral splint for temporomandibular joint disorders with revolutionary fluid system. Dent Res J 2013;10(3): 307-313.

19. Wieckiewicz M, Boening K, Wiland P, Shiau YY, Paradowska-Stolarz A. Reported concepts for the treatment modalities and pain management of temporomandibular disorders. J Headache Pain. 2015;16:106.

20. Kui A, Buduru S, lacob S, Manziuc M, Mitariu L. The use of occlusal splints in the management of temporomandibular disorder. Heal Sport Rehabil Med. 2020;21(2):82-87.

21. Piper MA. TMJ diagnostics and basic management. Seminar manual. Piper Clinic, St. Petersburg, FL. 2006.

22. Shankland WE. Nociceptive trigeminal inhibitiontension suppression system: a method of preventing migraine and tension headaches. Compend Contin Educ Dent. 2002;23:105-113.

23. Altindiş $T$, Güngörmüş $M$. Thermographic evaluation of occlusal splint and low level laser therapy in myofascial pain syndrome. Complement Ther Med. 2019;44:277-281.

24. Dalewski B, Chruściel-Nogalska M, Frączak B. Occlusal splint versus modified nociceptive trigeminal inhibition splint in bruxism therapy: a randomized, controlled trial using surface electromyography. Aust Dent J. 2015;60(4):445454.

25. Kuzmanovic Pficer J, Dodic S, Lazic V, Trajkovic G, Milic N, Milicic B. Occlusal stabilization splint for patients with temporomandibular disorders: Meta-analysis of short and long term effects. PLoS One. 2017;12(2):e0171296. doi:10.1371/journal. pone.0171296

26. Choudhary S, Rao HM, Rohilla AK, Cherenjeevi J. The Occlusal Splint Therapy: A Literature Review. Indian J Dent Sci. 2015;7(1):101-108.

27. 27. Buduru S, Talamaceanu D, Baru O, Buduru R, Szuhanek C, Mesaros A. CAD_CAM occlusal splints : milling and printing methods. Revista de Chimie. 2018;69(12):3461-3463.

\section{Corresponding author:}

Buduru Smaranda

Iuliu Hatieganu University of Medicine and Pharmacy, 32 Clinicilor Street, Cluj-Napoca, Romania

Email: smarandabudurudana@gmail.com 